\title{
The Differences in Sleep Skills between Insomnia Patients with and without Need of Hypnotics
}

\author{
Byunghoon Kong, MD ${ }^{1}$, Mi Ae Ko, MD¹, Jhin Goo Chang, MD¹, Minha Hong, MD, PhD¹, Woo Jung Kim, MD, PhD ${ }^{1,2}$, \\ Su Young Lee, MD, PhD¹, Hyunsoo Kim, MD¹, Hoo Rim Song, MD, PhD \\ 'Department of Psychiatry, Myongji Hospital, Hanyang University Medical Center, Goyang, Korea \\ 2Department of Psychiatry, Yongin Severance Hospital, Yonsei University College of Medicine, Yongin, Korea
}

\author{
Received: July 24, 2020 \\ Revised: September 1, 2020 \\ Accepted: September 10, 2020 \\ Correspondence \\ Hoo Rim Song, MD, PhD \\ Department of Psychiatry, Myongi Hospital, \\ Hanyang University Medical Center, \\ 55 Hwasu-ro 14beon-gil, Deogyang-gu, \\ Goyang 10475, Korea \\ Tel +82-31-810-5114 \\ Fax +82-31-969-0500 \\ E-maildrsong@empas.com \\ ORCID \\ Byunghoon Kong \\ https://orcid.org/0000-0003-1506-1706 \\ Mi Ae Ko \\ https://orcid.org/0000-0001-7730-5804 \\ Jhin Goo Chang \\ https://orcid.org/0000-0003-3024-4893 \\ Minha Hong \\ https://orcid.org/0000-0003-4924-1107 \\ Woo Jung Kim \\ https://orcid.org/0000-0002-4963-4819 \\ $\mathrm{Su}$ Young Lee \\ https://orcid.org/0000-0003-3125-3409 \\ Hyunsoo Kim \\ https://orcid.org/0000-0002-6219-8820 \\ Hoo Rim Song \\ https://orcid.org/0000-0001-8927-0530 \\ (c) This is an Open Access article distributed un- \\ der the terms of the Creative Commons Attribu- \\ tion Non-Commercial License (https://creative- \\ commons.org/licenses/by-nc/4.0) which permits \\ unrestricted non-commercial use, distribution, \\ and reproduction in any medium, provided the \\ original work is properly cited.
}

Background and Objective Hypnotics are widely prescribed for the treatment of insomnia; however, cognitive behavioral therapy for insomnia (CBT-I) is considered as a primary treatment. We checked ordinary sleep skills of insomnia patients and changes in sleep skills after CBT-I, and evaluated the difference in sleep skills between patients with and without need of hypnotics.

Methods A total of 131 outpatients who had visited our sleep clinic with insomnia were recruited. Enrollment criteria included at least 12 weeks of insomnia periods, less than 16 in Beck Depression Inventory score, and agreement to receive 9 sessions of CBT-I for 12 weeks. After 12 weeks of CBT-I, patients were divided into two groups: still in need and no need of hypnotics. Changes in sleep skills were compared between these groups.

Results Sleep hygiene, sleep stimulus-control skills, and sleep Visual Analogue Scales (VAS) were statistically improved after CBT-I (all, p < 0.001). Forty-six (35.1\%) patients did not need hypnotics anymore; however, 85 (64.9\%) still needed hypnotics after CBT-I. Sleep VAS $(25.26 \pm 8.52$ vs. $32.64 \pm 8.95, \mathrm{p}<0.001)$, practicing regular moderate exercise $(3.67 \pm 0.92$ vs. $2.76 \pm 1.06, \mathrm{p}=$ $0.030)$, and having a relaxing bedtime routine $(4.08 \pm 0.55 \mathrm{vs.} 2.76 \pm 0.76, \mathrm{p}<0.001)$ were different in the two groups.

Conclusions Among CBT-I skills, regular moderate exercise and a relaxing bedtime routine seem to be key components. For reducing chronic use of hypnotics, and better outcome of CBT-I, clinicians should focus more on these two components.

Sleep Med Res 2020;11(2):88-93

Key Words Cognitive bahavioral therapy, Insomnia, Hypnotics.

\section{INTRODUCTION}

Insomnia is a common complaint in primary care patients. In self-reports of sleep difficulty, a prevalence of $10-40 \%$ is observed. Major current insomnia was observed in approximately $10 \%$ of primary care patients [1]. Insomnia is associated with significant morbidity such as reduced quality of life, increased risk of psychiatric disorders, and higher risk of various physical diseases such as hypertension and diabetes [2].

As a treatment for insomnia, approximately $3 \%$ of adults use hypnotic medication [3]. Although hypnotic medications are effective in managing short-term insomnia, concerns have been raised about psychomotor and cognitive impairment, difficulties with discontinuation, and dependence liability due to long-term hypnotic use [4]. In older adults, there may be concerns about falls and hip fractures [5], motor vehicle accidents [6], and mortality [7], as well as concerns about undesirable drug interactions and polypharmacy effects.

Cognitive behavioral therapy for insomnia (CBT-I), which has minimal adverse effects, is considered the gold standard therapy in treatment of insomnia [8]. CBT-I can assist patients 
in integrating management skills for new learners with insomnia, and in stopping medication [9]. Evidence-based guidelines conclude that CBT-I not only outranks hypnotic treatment in the short term, but also in the long term concerning therapeutic efficiency [10]. Even if a combined CBT-I plus medication approach provided an added benefit immediately after treatment, extending CBT-I while tapering medication produces better sustained improvements compared to continued use of medication as needed [11]. Despite the strong evidence for efficacy, this kind of structured intervention may be time consuming and is not always readily accessible [12].

In many clinical trials, CBT-I is delivered in multi-component formats. This makes it difficult to identify critical ingredients responsible for improvement [13]. In particular, it is unclear which CBT-I elements are effective for people who do not need hypnotics.

In this study, we checked the ordinary sleep skills of insomnia patients, and changes in sleep skills after CBT-I, and evaluated the difference in changes in sleep skills between patients with and without need of hypnotics.

\section{METHODS}

\section{Participants}

This study was approved by the Institutional Review Board of Myongji Hospital, Goyang, Korea (Study No. NON2018-003008). Under the approval by the Ethics Committee, outpatients who had visited our sleep clinic suffering from insomnia without any psychiatric co-morbidities (primary insomnia according to the DSM-IV-TR criteria) were recruited. All patients provided written informed consent before entering this study, and their anonymity was preserved. The enrollment criteria included 1) at least 12 weeks of insomnia periods, 2) less than 16 in the Beck Depression Inventory (BDI) score, and 3) agreement to receive 9 sessions of CBT-I for 12 weeks.

\section{Measures}

Sleep disturbance was briefly assessed by "How much of a problem has sleeping been for you over the past week?" with 0-100 mm Visual Analogue Scales (VAS; sleep is no problem $=0$, and sleep is a severe problem $=100)$. All the patients had been receiving 9 sessions of CBT-I and were permitted to take hypnotic medications such as zolpidem or triazolam or other benzodiazepines for prn use for 12 weeks. CBT-I was focused on the understanding and application of sleep hygiene and sleep stimulus-control guidelines.

Sleep hygiene (SH) guidelines were as follows: limit the amount of time spent in bed and maintain a regular sleep/wake cycle (SH1), get regular moderate exercise in the late afternoon or early evening ( $\mathrm{SH} 2$ ), avoid exposure to bright light at night (SH3), avoid heavy meals or drinking within 3 hours of bedtime (SH4), enhance your environment by making sure the bedroom is quiet, dark, and comfortable (SH5), avoid caffeine, alcohol, and nicotine before bed (SH6), practice a relaxing bedtime routine ( $\mathrm{SH} 7$ ), have a light bedtime snack ( $\mathrm{SH} 8$ ), remove the bedroom clock (SH9). Sleep stimulus-control (SSC) guidelines were as follows: go to bed only when sleepy (SSC1), use the bed or bedroom only for sleeping or sex (SSC2), get out of bed when unable to sleep (SSC3), get up at the same time every morning (SSC4), avoid daytime napping (SSC5) [14].

The degree of each sleep skill state was evaluated using the Likert Scale (I could not do this strongly $=1$, I could not do this $=2$, Neutral $=3$, I could do this $=4$, I could do this strongly $=5$ ), and they were compared before and after CBT-I. After 12 weeks of CBT-I, patients were divided into two groups: still in need of hypnotics and no need of hypnotics. These two groups were compared in terms of changes in sleep skills.

\section{Data Analysis}

Statistical analyses were performed with the SPSS 24.0 (IBM Corp., Armonk, NY, USA). A chi-square test was used to compare the categorical variables between the two groups. Fisher's exact test was used when the expected frequency was more than $20 \%$. For the variables, excluding the categorical variables, the changes in measurement scores were compared using a paired $t$ test. The differences between the two groups, still in need of hypnotics and no need of hypnotics, were compared using a repeated measures analysis of variance. A p-value of $<0.05$ was considered statistically significant for all tests. All pairwise treatment comparisons utilized two-tailed tests.

\section{RESULTS}

A total of 131 patients were enrolled. The mean age was 48.35 years old, and 85 patients (64.9\%) were female. Forty-six (35.1\%) patients replied that they did not need hypnotics anymore; however, 85 (64.9\%) replied that they still needed hypnotics after CBT-I. There was no difference in the age, sex ratio, and comorbidities between the two groups. The patients had undergone a previous 25-week treatment for their sleep problems, and 122 patients (93.1\%) had used sleeping pills while 16 patients $(12.2 \%)$ had received CBT-I in the past. In terms of comorbidity, 38 patients (29.0\%) presented hypertension, 25 (19.1\%) presented dyslipidemia, and 23 (17.6\%) presented diabetes (Table 1).

The baseline sleep skill score of insomnia patients ranged from 2 (I could not do this) to 3 (Neutral) points, and the VAS score of sleep discomfort was 74.42 points. After 12 weeks of CBT-I, all the sleeping skill items were improved compared to those before CBT-I, and the VAS score of sleep discomfort (30.05 \pm 9.46 vs. $74.42 \pm 8.88, \mathrm{p}<0.001)$ was significantly lower than before CBT-I (all, p < 0.001) (Table 2).

However, even after CBT-I, those in need of hypnotics were 
Table 1. Baseline demographic and clinical characteristics

\begin{tabular}{|c|c|c|c|}
\hline Variables & Total $(\mathrm{n}=131)$ & $\begin{array}{c}\text { Still in need of hypnotics } \\
\text { after CBT-I }(\mathrm{n}=85)\end{array}$ & $\begin{array}{l}\text { No need of hypnotics } \\
\text { after CBT-I }(\mathrm{n}=46)\end{array}$ \\
\hline Age (years) & $48.35 \pm 14.06$ & $45.35 \pm 16.33$ & $47.55 \pm 12.34$ \\
\hline Female & $85(64.9)$ & $54(63.5)$ & $31(67.4)$ \\
\hline Prevalence period of insomnia (weeks) & $25.15 \pm 11.57$ & $27.25 \pm 15.23$ & $24.33 \pm 10.78$ \\
\hline Previous history of taking hypnotics & $122(93.1)$ & $80(94.1)$ & $42(91.3)$ \\
\hline Previous history of undergoing CBT-I & $16(12.2)$ & $10(11.8)$ & $6(13.0)$ \\
\hline \multicolumn{4}{|l|}{ Comorbid medical illness } \\
\hline Hypertension & $38(29.0)$ & $28(32.9)$ & $10(21.7)$ \\
\hline Dyslipidemia & $25(19.1)$ & $18(21.2)$ & $7(15.2)$ \\
\hline Diabetes & $23(17.6)$ & $15(17.6)$ & $8(17.4)$ \\
\hline Renal disorder & $7(5.3)$ & $5(5.9)$ & $2(4.3)$ \\
\hline Liver disorder & $12(9.2)$ & $8(9.4)$ & $4(8.7)$ \\
\hline Others & $24(18.3)$ & $20(23.5)$ & $4(8.7)$ \\
\hline \multicolumn{4}{|l|}{ Prescribed hypnotics (duplicate summation) } \\
\hline Zolpidem & $67(51.2)$ & $46(54.1)$ & $21(45.7)$ \\
\hline Triazolam & $5(3.8)$ & $4(4.7)$ & $1(2.2)$ \\
\hline Benzodiazepines & $59(45.0)$ & $38(44.7)$ & $21(45.7)$ \\
\hline Beck Depression Inventory & $9.62 \pm 2.59$ & $11.45 \pm 4.04$ & $8.84 \pm 1.09$ \\
\hline
\end{tabular}

Data are presented as $\mathrm{n}(\%)$ or mean \pm standard deviation.

CBT-I: cognitive behavioral therapy for insomnia.

nearly twice as likely as those who did not need hypnotics. Further, both groups, still in need of hypnotics and no need of hypnotics, displayed equal and significant improvement on the Likert Scale. Regarding comparing the degree of improvement in sleep skills, sleep VAS ( $25.26 \pm 8.52$ vs. $32.64 \pm 8.95, \mathrm{p}<0.001)$, $\mathrm{SH} 2(3.67 \pm 0.92$ vs. $2.76 \pm 1.06, \mathrm{p}=0.030)$, and SH7 (4.08 \pm 0.55 vs. $2.76 \pm 0.76, \mathrm{p}<0.001)$ were different in the two groups after CBT-I (Table 3). In the group of those who did not require hypnotics anymore, the above items were significant in the range of improvement.

\section{DISCUSSION}

This study focused on the implementation of sleeping skills through CBT-I for 12 weeks in patients with insomnia, and investigated whether there is a difference in sleep skills between patients with and without the need of hypnotics. The results showed that CBT-I has effects on improvement in sleep skills and self-rating sleep VAS scores. There was a difference in sleep skills between the two groups, and daytime regular exercise and relaxation therapy were found to be further improved in the group that terminated the drug after CBT-I.

In this study, before CBT-I, insomnia patients did not perform well in terms of sleep hygiene. This is consistent with previous studies that indicated that insomnia patients did not only have less sleep hygiene knowledge but their sleep hygiene practices were also worse compared to those of good sleepers [15]. In both groups, the sleep discomfort VAS scores decreased, and the degree of practice increased in terms of sleep hygiene after CBT-I. This indicates that obvious gaps in awareness and practice could be remedied by education and/or exhortation during behavioral treatment for insomnia [15].

This is a commonly used treatment; however, there is no absolute consensus as to what steps should be included to construct sleep hygiene practices. Further, there are overlaps between sleep hygiene and cognitive-behavioral treatments such as sleep stimulus-control and sleep restriction therapy. There were mixed results regarding the effects when individual components of sleep hygiene among normal sleepers were manipulated under experimental conditions. Therefore, the emphasis should be on the guidelines for the use of individual rules, rather than the integrated effects of items in sleep hygiene [16].

Our results show that CBT-I improves sleep skills, especially for those who do not need hypnotics and are better at maintaining regular daytime exercise and practicing nighttime relaxation.

Physical exercise is an effective intervention practice in improving the quantity or quality of sleep [17]. In a sample of adults included in a meta-analysis, it was suggested that exercise is associated with improvements in sleep outcome [18]. Further, given the possible side effects of hypnotics, exercise is an alternative treatment for older people as it may improve sleep and quality of life, although not for everyone [19]. In the above-mentioned studies, more than $80 \%$ of the participants were over 60 years old; however, the average age in our study was 48.35 years old. A high rate in body heating can persist for 1 to 2 hours, especial- 
Table 2. Sleep skills and state before and after CBT-I $(n=131)$

\begin{tabular}{lccc}
\hline & Before CBT-I & After CBT-I & Paired t test \\
\hline SH1 & $2.58 \pm 0.96$ & $3.48 \pm 0.77$ & $<0.001$ \\
SH2 & $2.16 \pm 1.09$ & $3.08 \pm 1.10$ & $<0.001$ \\
SH3 & $3.32 \pm 1.01$ & $4.08 \pm 0.64$ & $<0.001$ \\
SH4 & $3.41 \pm 1.09$ & $4.12 \pm 0.68$ & $<0.001$ \\
SH5 & $2.85 \pm 1.11$ & $3.89 \pm 0.75$ & $<0.001$ \\
SH6 & $3.22 \pm 1.16$ & $4.06 \pm 0.65$ & $<0.001$ \\
SH7 & $2.36 \pm 0.97$ & $3.22 \pm 0.94$ & $<0.001$ \\
SH8 & $3.10 \pm 0.91$ & $4.03 \pm 0.64$ & $<0.001$ \\
SH9 & $3.30 \pm 0.97$ & $4.49 \pm 0.59$ & $<0.001$ \\
SSC1 & $2.67 \pm 0.97$ & $3.85 \pm 0.68$ & $<0.001$ \\
SSC2 & $2.84 \pm 1.02$ & $3.81 \pm 0.78$ & $<0.001$ \\
SSC3 & $2.38 \pm 0.99$ & $3.13 \pm 0.87$ & $<0.001$ \\
SSC4 & $2.58 \pm 1.18$ & $3.34 \pm 0.73$ & $<0.001$ \\
SSC5 & $2.90 \pm 1.16$ & $4.01 \pm 0.75$ & $<0.001$ \\
Sleep VAS & $74.42 \pm 8.88$ & $30.05 \pm 9.46$ & $<0.001$ \\
\hline Data
\end{tabular}

Data are presented as mean \pm standard deviation. $\mathrm{SH} 1$ : limit the amount of time spent in bed and maintain a regular sleep/wake cycle, $\mathrm{SH}$ 2: get regular moderate exercise in the late afternoon or early evening, SH3: avoid exposure to bright light at night, SH4: avoid heavy meals or drinking within 3 hours of bedtime, SH5: enhance your environment by making sure the bedroom is quiet, dark, and comfortable, SH6: avoid caffeine, alcohol, and nicotine before bed, SH7: practice a relaxing bedtime routine, SH8: have a light bedtime snack, SH9: remove the bedroom clock, SSC1: go to bed only when sleepy, SSC2: use the bed or bedroom only for sleeping or sex, SSC3: get out of bed when unable to sleep, SSC4: get up at the same time every morning, SSC5: avoid daytime napping. SH: sleep hygiene, SSC: sleep stimulus-control, VAS: Visual Analogue Scales (0-100 mm), CBT-I: cognitive behavioral therapy for insomnia.

ly a rapid rise in core temperature and in the rate of dehydration can cause slow-wave sleep. Exercise can be the means to do just that. After intense exercise, the core body temperature increases, and after 4-6 hours, a compensatory decrease in body temperature occurs [20]. Therefore, if you do aerobic exercise with a certain degree of intensity for about 20 minutes in the late afternoon or early evening, rebound cooling occurs at around midnight, which can help you get better sleep [21].

To reduce high levels of arousal, patients are taught how to apply relaxation therapies. Relaxation training includes autogenic training, imagery training, hypnosis, meditation, yoga, abdominal breathing, progressive muscle relaxation, and visual or auditory biofeedback therapy. Each of these assists by recalling an environment with a comfortable body perception, focusing on a pleasing image, relaxing from the feet to the facial muscles, or instructing certain physiological factors to be controlled [22]. Whatever the origin, a high level of tension in the gross skeletal muscles is observed in insomnia, so relaxation therapy is helpful [23].
In previous studies in the Canadian primary care setting, all sleep and mood measures improved after the CBT-I program. After CBT-I, the frequency of sleep medication was also reduced [24]. In other systematic literature reviews, CBT-I has been indicated to be effective in the treatment of depression comorbid with insomnia because an improvement in insomnia symptoms as caused by CBT-I leads to a decrease in depression [25]. However, we only recruited participants with a BDI lower than 16 and did not evaluate the effects of CBT-I on mood.

There were differences in sleep skills and sleep discomfort VAS scores between the groups after CBT-I. The practice of the above items was more effective in the group that did not need hypnotics. These results support that the best response and remission rates were for individuals who were initially treated with combined therapy but then switched to CBT-I alone during the extended treatment phase [26].

In this study, differences in the sleep skills between the two groups showed variances in the application of regular daytime exercise and relaxation therapies. Previous studies have been conducted on the differences in sleep skills. In a previous study, when assessing sleep hygiene knowledge and practice, insomniacs most often reported that they do not have regular exercise habits, they worry as they prepare for bed, and they do not set aside a nighttime period to relax [15]. In another study conducted in Korea, the items of exercise and relaxation were not wellpracticed in patients with insomnia; however, the performance of these items was reported to be very effective for sleep [27].

Progressive relaxation therapies and regular daytime exercise are well-established behavioral treatments for insomnia [28]. When employed during pre-sleep periods, these therapies are presumed to counteract the cognitive and physiologic mechanisms that initiate and perpetuate insomnia [29]. Exercise improves subjective sleep satisfaction. Exercise training has a moderate beneficial effect on sleep quality and the subdomains of subjective sleep quality, sleep latency, and sleep medication usage [30].

The efficacy of the different components of CBT-I as standalone interventions has been rarely investigated or compared [10]. In this study, regular moderate exercise during daytime and a relaxing bedtime routine seem to be key components. For reducing chronic use of hypnotics, and for better outcome of CBTI, clinicians should focus more on these two components. To improve sleep skills, reminders can be used to improve adherence and the outcome of interventions as people are rarely aware of their sleep behavior, and the monitoring of progress can be easily missed. Reminding people to do their relaxation exercises improves adherence to target behaviors and provides an opportunity to test the effect [31].

There were several limitations to have definite interpretations; this study was conducted with a small number of patients in a single center, and results were based on the self-rating questionnaire. In addition, we evaluated only short-term prognosis after 
Table 3. Difference in sleep skills and state between patients with and without need of hypnotics after CBT-I

\begin{tabular}{|c|c|c|c|c|c|c|c|c|c|}
\hline & \multicolumn{3}{|c|}{ Still in need of hypnotics after CBT-I $(\mathrm{n}=85)$} & \multicolumn{3}{|c|}{ No need of hypnotics after CBT-I $(\mathrm{n}=46)$} & \multirow{2}{*}{$\begin{array}{c}\text { Student } \\
\text { t test (before } \\
\text { CBT-I) }\end{array}$} & \multirow{2}{*}{$\begin{array}{c}\text { Student } \\
\text { t test (after } \\
\text { CBT-I) }\end{array}$} & \multirow{2}{*}{$\begin{array}{c}\text { Repeated } \\
\text { measures } \\
\text { ANOVA }\end{array}$} \\
\hline & Before CBT-I & After CBT-I & $\begin{array}{c}\text { Paired } \\
\mathrm{t} \text { test }\end{array}$ & Before CBT-I & After CBT-I & $\begin{array}{c}\text { Paired } \\
\mathrm{t} \text { test }\end{array}$ & & & \\
\hline SH1 & $2.61 \pm 0.98$ & $3.48 \pm 0.78$ & $<0.001$ & $2.54 \pm 0.93$ & $3.50 \pm 0.78$ & $<0.001$ & 0.701 & 0.902 & 0.854 \\
\hline $\mathrm{SH} 2$ & $2.21 \pm 1.09$ & $2.76 \pm 1.06$ & $<0.001$ & $2.06 \pm 1.10$ & $3.67 \pm 0.92$ & $<0.001$ & 0.466 & $<0.001$ & $0.030^{*}$ \\
\hline $\mathrm{SH} 3$ & $3.30 \pm 1.00$ & $4.04 \pm 0.68$ & $<0.001$ & $3.36 \pm 1.06$ & $4.15 \pm 0.55$ & $<0.001$ & 0.734 & 0.375 & 0.178 \\
\hline SH4 & $3.31 \pm 1.07$ & $4.09 \pm 0.71$ & $<0.001$ & $3.58 \pm 1.12$ & $4.19 \pm 0.61$ & $<0.001$ & 0.180 & 0.419 & 0.176 \\
\hline SH5 & $2.82 \pm 1.15$ & $3.78 \pm 0.75$ & $<0.001$ & $2.91 \pm 1.05$ & $4.08 \pm 0.72$ & $<0.001$ & 0.663 & 0.031 & 0.194 \\
\hline SH6 & $3.16 \pm 1.15$ & $4.00 \pm 0.65$ & $<0.001$ & $3.34 \pm 1.19$ & $4.19 \pm 0.65$ & $<0.001$ & 0.393 & 0.105 & 0.193 \\
\hline SH7 & $2.34 \pm 0.97$ & $2.76 \pm 0.76$ & $<0.001$ & $2.41 \pm 1.00$ & $4.08 \pm 0.55$ & $<0.001$ & 0.690 & $<0.001$ & $<0.001^{*}$ \\
\hline SH8 & $3.08 \pm 0.88$ & $4.02 \pm 0.67$ & $<0.001$ & $3.15 \pm 0.96$ & $4.06 \pm 0.61$ & $<0.001$ & 0.678 & 0.727 & 0.642 \\
\hline SH9 & $3.29 \pm 0.94$ & $4.47 \pm 0.64$ & $<0.001$ & $3.32 \pm 1.03$ & $4.54 \pm 0.50$ & $<0.001$ & 0.859 & 0.509 & 0.650 \\
\hline SSC1 & $2.67 \pm 0.99$ & $3.77 \pm 0.67$ & $<0.001$ & $2.69 \pm 0.96$ & $4.00 \pm 0.66$ & $<0.001$ & 0.889 & 0.073 & 0.332 \\
\hline SSC2 & $2.82 \pm 1.07$ & $3.77 \pm 0.74$ & $<0.001$ & $2.89 \pm 0.94$ & $3.89 \pm 0.84$ & $<0.001$ & 0.720 & 0.425 & 0.511 \\
\hline SSC3 & $2.37 \pm 0.98$ & $2.96 \pm 0.85$ & $<0.001$ & $2.39 \pm 1.02$ & $3.45 \pm 0.83$ & $<0.001$ & 0.936 & 0.002 & 0.078 \\
\hline SSC4 & $2.60 \pm 1.19$ & $3.36 \pm 0.78$ & $<0.001$ & $2.54 \pm 1.18$ & $3.30 \pm 0.62$ & $<0.001$ & 0.796 & 0.654 & 0.696 \\
\hline SSC5 & $2.88 \pm 1.18$ & $4.03 \pm 0.79$ & $<0.001$ & $2.95 \pm 1.13$ & $4.01 \pm 0.75$ & $<0.001$ & 0.730 & 0.681 & 0.953 \\
\hline Sleep VAS & $74.45 \pm 8.97$ & $32.64 \pm 8.95$ & $<0.001$ & $74.36 \pm 8.83$ & $25.26 \pm 8.52$ & $<0.001$ & 0.957 & $<0.001$ & $0.005^{*}$ \\
\hline
\end{tabular}

Data are presented as mean \pm standard deviation. SH1: limit the amount of time spent in bed and maintain a regular sleep/wake cycle, $\mathrm{SH} 2$ : get regular moderate exercise in the late afternoon or early evening, SH3: avoid exposure to bright light at night, SH4: avoid heavy meals or drinking within 3 hours of bedtime, SH5: enhance your environment by making sure the bedroom is quiet, dark, and comfortable, SH6: avoid caffeine, alcohol, and nicotine before bed, SH7: practice a relaxing bedtime routine, SH8: have a light bedtime snack, SH9: remove the bedroom clock, SSC1: go to bed only when sleepy, SSC2: use the bed or bedroom only for sleeping or sex, SSC3: get out of bed when unable to sleep, SSC4: get up at the same time every morning, SSC5: avoid daytime napping.

${ }^{*} \mathrm{p}<0.05$ in repeated measures ANOVA.

SH: sleep hygiene, SSC: sleep stimulus-control, VAS: Visual Analogue Scales (0-100 mm), CBT-I: cognitive behavioral therapy for insomnia, ANOVA: analysis of variance.

CBT-I. The impact of prescribed hypnotics on outcomes was not evaluated. Also, no assessment was made of the effects of comorbid medical illness on outcomes. Finally, focusing on sleep techniques as measurable indicators might be insufficient to represent the entire CBT-I components.

Despite these limitations, our research is meaningful in the identification of important elements of CBT-I. In addition, daytime exercise and relaxation techniques were identified through the sleep techniques of patients who did not take hypnotic medications after CBT-I. How about focusing more on CBT-I and on these two skills? Further studies are needed to elucidate the impact factors and long-term effects of CBT-I with larger, more sophisticated designs.

\section{Acknowledgments}

We would like to thank Editage (www.editage.co.kr) for English language editing.

\section{Conflicts of Interest}

The authors have no financial conflicts of interest.

\section{Authors' Contribution}

Conceptualization: Ko MA, Chang JG, Hong M, Kim WJ, Lee SY, Kim H, Song HR. Formal analysis: Kong B, Ko MA, Song HR. Writing_original draft: Kong B, Ko MA, Song HR. Writing—review \& editing: Song HR.

\section{REFERENCES}

1. Simon GE, VonKorff M. Prevalence, burden, and treatment of insomnia in primary care. Am J Psychiatry 1997;154:1417-23.

2. Foley D, Ancoli-Israel S, Britz P, Walsh J. Sleep disturbances and chronic disease in older adults: results of the 2003 National Sleep Foundation Sleep in America Survey. J Psychosom Res 2004;56:497-502.

3. Mellinger GD, Balter MB, Uhlenhuth EH. Insomnia and its treatment. Prevalence and correlates. Arch Gen Psychiatry 1985;42:225-32.

4. Roth T, Roehrs T, Vogel G. Zolpidem in the treatment of transient insomnia: a double-blind, randomized comparison with placebo. Sleep 1995; 18:246-51.

5. Ray WA, Griffin MR, Schaffner W, Baugh DK, Melton LJ 3rd. Psychotropic drug use and the risk of hip fracture. N Engl J Med 1987;316: 363-9.

6. Hemmelgarn B, Suissa S, Huang A, Boivin JF, Pinard G. Benzodiazepine use and the risk of motor vehicle crash in the elderly. JAMA 1997; 278:27-31.

7. Kripke DF, Klauber MR, Wingard DL, Fell RL, Assmus JD, Garfinkel L. Mortality hazard associated with prescription hypnotics. Biol Psychiatry 1998;43:687-93.

8. Sivertsen B, Omvik S, Pallesen S, Bjorvatn B, Havik OE, Kvale G, et al. Cognitive behavioral therapy vs zopiclone for treatment of chronic primary insomnia in older adults: a randomized controlled trial. JAMA 2006;295:2851-8. 
9. Morin CM, Colecchi C, Stone J, Sood R, Brink D. Behavioral and pharmacological therapies for late-life insomnia: a randomized controlled trial. JAMA 1999;281:991-9.

10. Riemann D, Baglioni C, Bassetti C, Bjorvatn B, Dolenc Groselj L, Ellis JG, et al. European guideline for the diagnosis and treatment of insomnia. J Sleep Res 2017;26:675-700.

11. Beaulieu-Bonneau S, Ivers H, Guay B, Morin CM. Long-term maintenance of therapeutic gains associated with cognitive-behavioral therapy for insomnia delivered alone or combined with zolpidem. Sleep 2017; 40:zsx002.

12. Wu R, Bao J, Zhang C, Deng J, Long C. Comparison of sleep condition and sleep-related psychological activity after cognitive-behavior and pharmacological therapy for chronic insomnia. Psychother Psychosom 2006; 75:220-8.

13. Harvey L, Inglis SJ, Espie CA. Insomniacs' reported use of CBT components and relationship to long-term clinical outcome. Behav Res Ther 2002;40:75-83.

14. Perlis ML, Jungquist C, Smith MT, Posner D. Cognitive behavioral treatment of insomnia: a session-by-session guide. New York, NY: SpringerVerlag New York 2005.

15. Lacks P, Rotert M. Knowledge and practice of sleep hygiene techniques in insomniacs and good sleepers. Behav Res Ther 1986;24:365-8.

16. Stepanski EJ, Wyatt JK. Use of sleep hygiene in the treatment of insomnia. Sleep Med Rev 2003;7:215-25.

17. Dolezal BA, Neufeld EV, Boland DM, Martin JL, Cooper CB. Interrelationship between sleep and exercise: a systematic review. Adv Prev Med 2017;2017:1364387.

18. Kelley GA, Kelley KS. Exercise and sleep: a systematic review of previous meta-analyses. J Evid Based Med 2017;10:26-36.

19. Montgomery P, Dennis J. Physical exercise for sleep problems in adults aged 60+. Cochrane Database Syst Rev 2002;2002:CD003404.

20. Horne JA, Staff LH. Exercise and sleep: body-heating effects. Sleep 1983;
6:36-46

21. Hauri PJ. Sleep hygiene, relaxation therapy, and cognitive interventions. In: Hauri PJ. Case studies in insomnia. Boston, MA: Springer 1991.

22. Maness DL, Khan M. Nonpharmacologic management of chronic insomnia. Am Fam Physician 2015;92:1058-64.

23. Kahn M, Baker BL, Weiss JM. Treatment of insomnia by relaxation training. J Abnorm Psychol 1968;73:556-8.

24. Davidson JR, Dawson S, Krsmanovic A. Effectiveness of group cognitive behavioral therapy for insomnia (CBT-I) in a primary care setting. Behav Sleep Med 2019;17:191-201.

25. Cunningham JEA, Shapiro CM. Cognitive behavioural therapy for insomnia (CBT-I) to treat depression: a systematic review. J Psychosom Res 2018;106:1-12.

26. Morin CM, Vallières A, Guay B, Ivers H, Savard J, Mérette C, et al. Cognitive behavioral therapy, singly and combined with medication, for persistent insomnia: a randomized controlled trial. JAMA 2009; 301:2005-15.

27. Choi YK, Kim L, Suh KY, Shin DK. A study on the practice and effects of sleep hygiene. Sleep Med Psychophysiol 1997;4:156-63.

28. Lichstein KL, Riedel BW. Behavioral assessment and treatment of insomnia: a review with an emphasis on clinical application. Behav Ther 1994;25:659-88.

29. Morgenthaler T, Kramer M, Alessi C, Friedman L, Boehlecke B, Brown T, et al. Practice parameters for the psychological and behavioral treatment of insomnia: an update. an american academy of sleep medicine report. Sleep 2006;29:1415-9.

30. Yang PY, Ho KH, Chen HC, Chien MY. Exercise training improves sleep quality in middle-aged and older adults with sleep problems: a systematic review. J Physiother 2012;58:157-63.

31. Horsch C, Spruit S, Lancee J, van Eijk R, Beun RJ, Neerincx M, et al. Reminders make people adhere better to a self-help sleep intervention. Health Technol (Berl) 2017;7:173-88. 